Draft Version OCtOBER 1, 2018

Preprint typeset using $\mathrm{LATEX}_{\mathrm{E}}$ style emulateapj v. 4/9/03

\title{
THE STAR FORMATION HISTORY OF THE DISK OF THE STARBURST GALAXY M 82
}

\author{
Y. D. Mayya ${ }^{1}$, A. Bressan ${ }^{1,2,3}$, L. Carrasco ${ }^{1}$, and L. Hernandez-Martinez ${ }^{4}$ \\ Draft version October 1, 2018
}

\begin{abstract}
Spectroscopic, photometric and dynamical data of the inner $3 \mathrm{kpc}$ part of the starburst galaxy M 82 are analyzed in order to investigate the star formation history of its disk. The long-slit spectra along the major axis are dominated by Balmer absorption lines in the region outside the nuclear starburst all the way up to $\approx 3.5$ scalelengths $\left(\mu_{B}=22 \mathrm{mag} \operatorname{arcsec}^{-2}\right)$. Single Stellar Population (SSP) spectra of age 0.4-1.0 Gyr match well the observed spectra in the $1-3 \mathrm{kpc}$ zone, with a mean age of the stellar population marginally higher in the outer parts. The mass in these populations, along with that in the gas component, make up for the inferred dynamical mass in the same annular zone for a Kroupa initial mass function, with a low mass cut-off $m_{l}=0.4 \mathrm{M}_{\odot}$. The observed ratio of the abundances of $\alpha$ elements with respect to $\mathrm{Fe}$, is also consistent with the idea that almost all the stars in M 82 disk formed in a burst of short duration (0.3 Gyr) around 0.8 Gyr ago. We find that the optical/near infrared colors and their gradients in the disk are determined by the reddening with visual extinction exceeding $1 \mathrm{mag}$ even in the outer parts of the disk, where there is apparently no current star formation. The disk-wide starburst activity was most likely triggered by the interaction of M 82 with its massive neighbor M 81 around 1 Gyr ago. The properties of the disk of M 82 very much resemble the properties of the disks of luminous compact blue galaxies seen at $0.2-1.0$ redshift. Subject headings: galaxies: individual (M 82) — galaxies: evolution — galaxies: interactions
\end{abstract}

\section{INTRODUCTION}

M82 is a nearby edge-on galaxy $(\mathrm{D}=3.63 \mathrm{Mpc}$, image scale $17.6 \mathrm{pc} \operatorname{arcsec}^{-1}$; Freedman et al. 1994), which harbors a nuclear starburst, the prototype of the starburst phenomenon (Rieke et al. 1980; Rieke et al. 1993). The optical appearance of M 82 is dominated by bright star-forming knots interspersed by dusty filaments (O'Connell \& Mangano 1978). This appearance has led to an Irr II morphological classification of M 82 (Holmberg 1950). A near-infrared (NIR) bar of $\sim 1 \mathrm{kpc}$ length was discovered by Telesco et al. (1991). Recently, Mavva. Carrasco \& Luna (2005) have discovered two symmetric spiral arms, suggesting a morphological classification SBc. Embedded inside the 500 pc starburst region is a small bulge, with an upper mass limit of $3 \times 10^{7} \mathrm{M}_{\odot}$ (Gaffnev. Lester \& Telesco 1993). The $K$-band scalelength of the disk is as small as 860 pc (Ichikawa et al. 1995).

M 82 is a low mass galaxy $\left(\sim 10^{10} \mathrm{M}_{\odot}\right)$, with a strong central concentration of mass and hardly any evidence for the dark matter halo (Sofue 1998). This mass is in the lower mass range of late-type galaxies. However, the observed luminosity, mean surface brightness and metallicity are higher than expected for a late-type galaxy. In addition, the percentage of mass in the gaseous component is as much as $\sim 50 \%$, which is abnormally high for a normal galaxy (Young \& Scoville 1984). Several authors have attributed these abnormalities to some phe-

\footnotetext{
1 Instituto Nacional de Astrofisica, Optica y Electronica, Luis Enrique Erro 1, Tonantzintla, C.P. 72840, Puebla, Mexico

2 Osservatorio Astronomico di Padova, Vicolo dell'Osservatorio 535122, Padova, Italy

3 SISSA, via Beirut 4, 34014, Trieste, Italy

4 Insituto de Astronómia, Universidad Nacional Autonoma de México, Apdo. Postal 70-264, Cd. Unversitaria 04510 Mxico DF, Mexico

Electronic address: ydm@inaoep.mx
}

nomenon associated with its interaction with other members of the M 81 group. Sofue (1998) explained the small size of the disk and the falling rotation curve with a model wherein M 82 lost its outer disk and halo during a closeby interaction with M81 and NGC 3077, which started around 1 Gyr ago. Yun. Ho. \& Lo (1994) reproduced the observed structure of the HI streamers using a numerical simulation, where M 82 is interacting with M 81 and NGC 3077. The closest approach occurred around 0.3 Gyr ago. Elvius (1972) presented a picture wherein M 82 captured a gas cloud during its passage in the M81 group, in order to explain the high gas fraction. Are the observed properties in the disk of M 82, especially, its high surface brightness, high metallicity, and low mass-to-light ratios, consistent with these scenarios? In this paper, we investigate the star formation history ( $\mathrm{SFH}$ ) that is consistent with the observed properties of the stellar disk, and compare the resultant SFH with phenomena that may be related to the interaction.

In Section 2, we describe the spectroscopic and imaging observations used in this work. Section 3 deals with a discussion of the quantities that can be used as constraints to the age of the stellar populations in the disk of M 82. In Section 4, we discuss the star formation and chemical evolutionary histories that are consistent with our data. The evolutionary status of M 82 is discussed in Section 5. A summary of the main results of this work is given in Section 6 .

\section{OBSERVATIONS AND ANALYSIS}

\subsection{Spectroscopic Observations}

Spectroscopic observations of M 82 were carried out with the purpose of registering the absorption-line dominated stellar spectra at a variety of radial distances from the center. Long-slit ( $3^{\prime}$ in length) spectra were taken at three positions along the major axis of the galaxy (P.A. of the slit $=62^{\circ}$ ) using the Boller \& Chivens spectro- 
graph at the Guillermo Haro Astrophysical Observatory, Cananea, Mexico. The observations were carried out on three nights, one in 1999 February and the other two in 2003 November. The starburst nucleus was placed at the center, extreme southwest and extreme northeast of the slit, respectively on the three nights. With the three slit positions, spectra up to a major axis distance of $3^{\prime}$ were registered on either side of the nucleus. At this radial distance, $B$-band surface brightness levels correspond to $22 \mathrm{mag}_{\operatorname{arcsec}}{ }^{-2}$. Two spectra, each of 30 minute duration, were taken for the bright central part, whereas three 30-minute spectra, were taken for the other two slit positions. A slit width of $1.6^{\prime \prime}$ was employed. A grating of 150 lines $/ \mathrm{mm}$ was used which covers a wavelength range of $\approx 3500-6800 \AA$ at a spectral resolution of around $10 \AA$. The spectral and spatial samplings were $3.2 \AA /$ pix and $0.46^{\prime \prime} /$ pix respectively. Object was observed as close to the meridian as possible, with maximum airmass of $\approx 1.30$. The instrumental response was calibrated by the observation of standard stars HR 1544, HR 5501, BD+40 4032, Feige 15 and Feige 34.

Each frame was bias-corrected and divided by a normalized flat-field using the tasks in the IRAF package. Wavelength-calibrated frames of the same slit position were averaged, in the process removing cosmic ray events. The disk of M 82 occupies the entire slit-length and hence sky spectrum could not be extracted from the same spectrum as the object. Instead, sky spectra were extracted from the spectra of galaxies with a compact nucleus observed immediately before and after the M82 observations.

\subsection{NIR and Optical Images}

NIR images in the $J, H$ and $K$ bands used in the present work were taken with the CAnanea Near Infrared CAmera (CANICA) ${ }^{5}$ available at the $2.1-\mathrm{m}$ telescope of the Observatorio Astrofísico Guillermo Haro in Cananea, Sonora, Mexico. Details of these images and the analysis procedure adopted were described in Mavva, Carrasco \& Luna (2005). Optical images in the $U, B, V$ and $R$ bands are based on the data archived at the NASA Extragalactic Database. These images were taken at the 40-inch telescope of the Mt. Laguna Observatory and were described by Marcum et al. (2001).

\section{CONSTRAINTS ON THE DISK PARAMETERS FROM OBSERVATIONAL DATA}

In this section, we combine our spectroscopic and photometric data with published dynamical data to put strong constraints on critical parameters of the stellar disk of M 82 .

\subsection{Spectroscopic ages of the stellar populations}

Two-dimensional spectra of M 82 were closely examined to isolate all regions with noticeably distinct surface brightness values. On either side of the starburst nucleus, there are bright continuum emitting knots up to around $60^{\prime \prime}(1 \mathrm{kpc})$ distance (e.g. region $\mathrm{B}$ on the northeast and region $\mathrm{G}$ on the southwest as defined by O'Connell \& Mangano 1978). Spectra representative of these continuum knots were extracted on the northeast

\footnotetext{
5 see http://www.inaoep.mx/ astrofi/cananea/canica/
}

and southwest directions separately. Beyond a radius of $1 \mathrm{kpc}$ on either side of the nucleus, bright knots are absent, and the spectra represent that of the underlying smooth disk. We defined two spectra on either side of the nucleus corresponding to radial zones between $1-2 \mathrm{kpc}$, and $2-2.7 \mathrm{kpc}$. The positions along the major axis of the extracted spectra are identified in Figure 1 by vertical hatches, which are labeled by their mean distances in arcsecs from the starburst nucleus. In this figure, we also show the major axis intensity cuts in the optical and NIR bands (solid lines). The exponential disk profiles (obtained by fitting the azimuthally averaged intensity profiles) are also plotted in this figure (dashed lines). The bottom two panels show the major axis color profiles. The dashed vertical lines mark the position of brightest region of the recently-discovered spiral arms, where the colors are marginally bluer. It is easily noticeable that colors become systematically redder towards the center of the galaxy.

The most critical part of the spectrum for population synthesis analysis is the blue part of the spectrum, because of the presence of a variety of spectral features sensitive to young, intermediate-age and old populations. This part of the extracted spectra are plotted in Figure 2. The signal-to-noise ratio of these spectra are good enough to measure spectral indices sensitive to age and metallicity. Some of the important absorption lines are marked in the figure. In general, all spec-

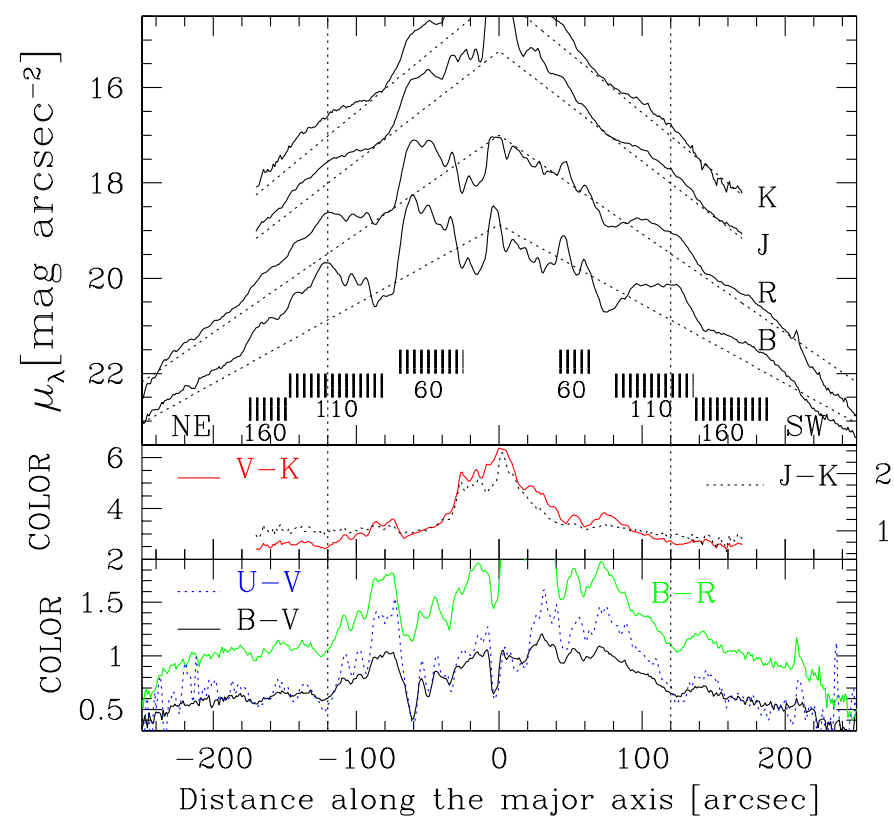

FIG. 1.- One-dimensional intensity and color profiles of M 82 . In the top panel, we show the intensity cuts along the major axis in $K, J, R$, and $B$ bands (solid lines), along with the exponential function that fits best the azimuthally averaged intensity profiles in the corresponding bands. The positions of the extracted spectra are shown by vertical grids, with the associated numbers denoting the mean distance in arcsecs of extracted regions from the nucleus. The NE and SW directions are also shown. The bottom two panels depict the major axis color profiles. The vertical dotted lines denote the intersection of the spiral arm with the major axis. 


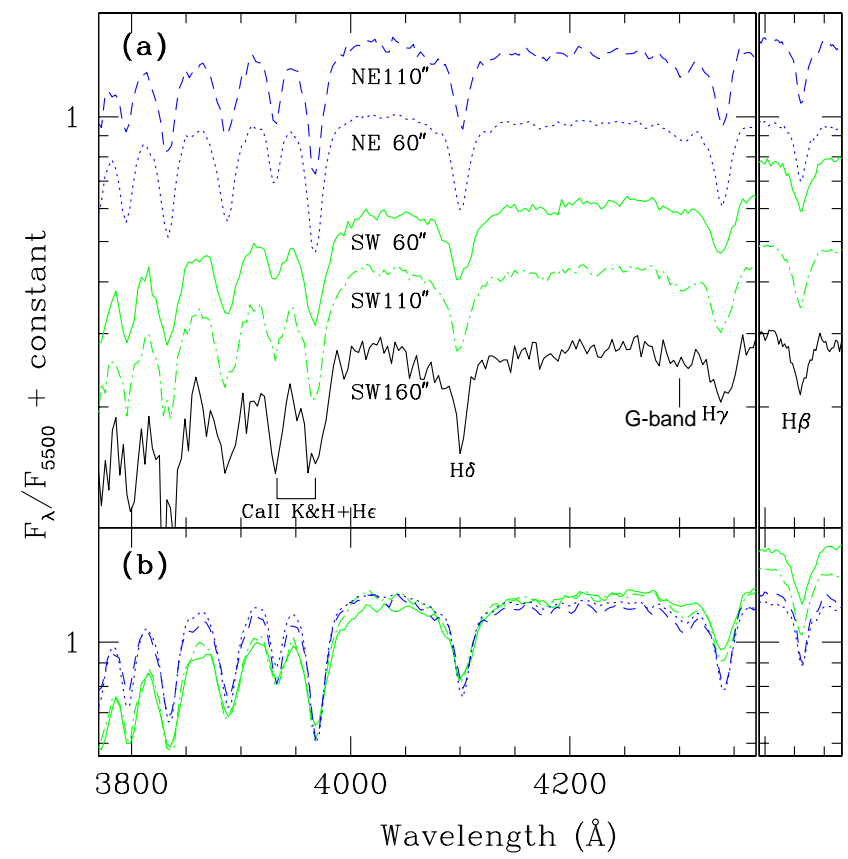

FIG. 2.- Major-axis spectra of the stellar disk of M 82 at five selected zones. In (a), the top two spectra correspond to the northeast (NE), and the bottom three correspond to the southwest (SW) part of the disk. The numbers appearing after the NE or SW correspond to the radial distance from the nucleus in arcsecs of the extracted spectra. Prominent age-sensitive absorption lines are identified. Note that the almost feature-less part of the spectra between $\mathrm{H} \gamma$ and $\mathrm{H} \beta$ is not shown. (b) The $60^{\prime \prime}$ and $110^{\prime \prime}$ spectra from either side are superposed (line types and colors the same as in panel a). Note that the strengths of all the absorption features in the four spectra are nearly identical, despite the NE spectra being systematically bluer than the SW spectra.

tra are dominated by Balmer absorption lines, which is consistent with the "A-F" spectral type inferred by O'Connell \& Mangano (1978). Relative depths of the spectral lines in the four spectra corresponding to the regions in the inner disk are identical. This is illustrated in panel (b) of the figure where these four spectra are superposed. The only difference is in the continuum shape, with the NE spectra systematically bluer than the SW spectra. In comparison, the spectrum of the outer disk $\left(\mathrm{SW} 110^{\prime \prime}\right)$ departs from the inner disk spectra in having deeper $\mathrm{Ca}$ II $\mathrm{K}$ line as compared to the $\mathrm{Ca} \mathrm{II} \mathrm{H}+\mathrm{H} \epsilon$ and other Balmer lines.

In the remaining part of this section, we use the observed spectra to infer the age of the stellar populations that give rise to these features, using model spectra for Single Stellar Populations (SSPs), properly smoothed to the observed resolution. The model SSPs have a Kroupa like initial mass function (IMF) — with masses between 0.15 and $120 \mathrm{M}_{\odot}$ (Kroupa et al. 1993). They are based on the Padova (Bressan et al. 1993; Fagotto et al. 1994a b) evolutionary tracks and on the Jacobv et al. (1984) library of stellar spectra. More details of the model can be found in Bressan et al. (1998). It is important to stress here that while we compare observed spectra with SSP models computed with the usual Kroupa IMF, in Sec. 4 we will discuss also a top-heavy modifica-

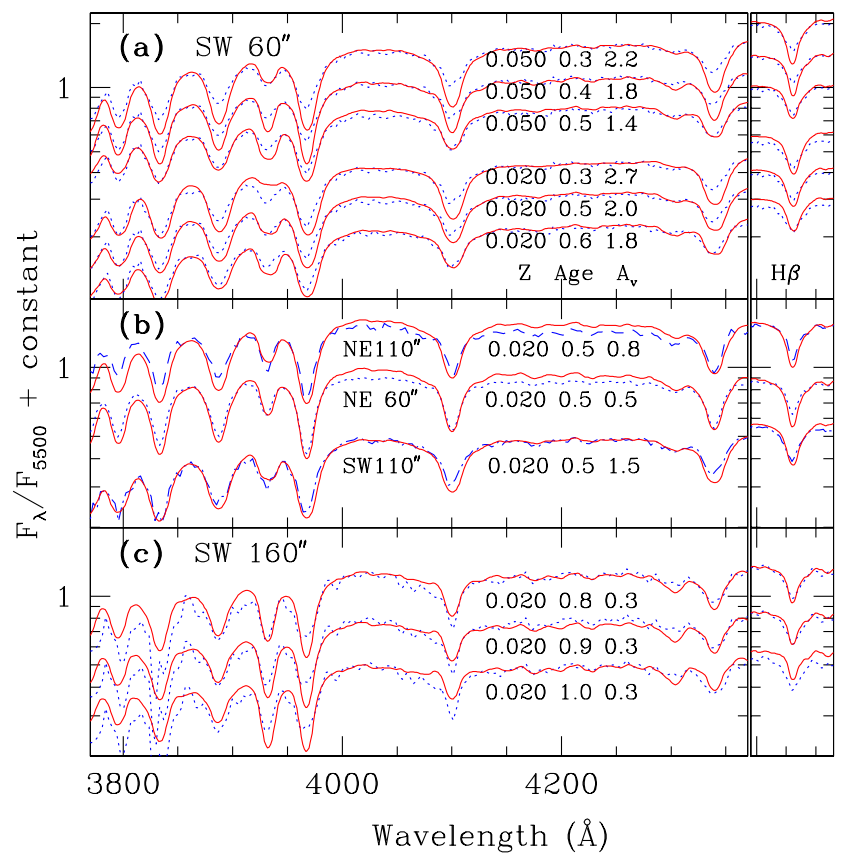

FIG. 3.- Observed spectra (dotted blue line) compared with model SSP spectra (solid red line). (a) Six models are superposed on the observed SW60" spectrum. The models correspond to two metallicities $(\mathrm{Z}=0.050,0.020)$, and three ages (0.3-0.6 Gyr). The best-match is obtained for $0.5 \mathrm{Gyr}$ at $\mathrm{Z}=0.020$. (b) The bestmatch model SSP superposed on the rest of the inner disk spectra. All these three spectra are consistent with an SSP of 0.5 Gyr at $\mathrm{Z}=0.020$, with the only difference between the spectra being the amount of reddening. (c) Three models are superposed on the SW $160^{\prime \prime}$ spectrum. This spectrum differs from the inner disk spectra in having the age-sensitive Ca II K profile almost as deep as the $\mathrm{Ca} \mathrm{II} \mathrm{H}+\mathrm{H} \epsilon$ profile, an indication of relatively older population. The model that best matches the important observed features is at $\mathrm{Z}=0.020$ for $0.9 \mathrm{Gyr}$.

tion of the IMF. The use of such a top-heavy IMF does not change our estimate of the age of the disk of M82.

Reddened model spectra are superposed on the observed spectrum for the SW60" zone in Figure 3a, where we have made use of Cardelli et al. (1989) reddening curve. The metallicity, age and the visual extinction of each model is indicated below each displayed spectrum. The intensity ratios of different features in the observed spectrum of the inner disk can be reproduced only for ages between 0.3 and $0.6 \mathrm{Gyr}$, with the exact age depending on the choice of metallicity. For ages younger than $0.3 \mathrm{Gyr}$, the Ca II $\mathrm{K}$ line does not appear in the SSP, whereas for older ages it is much stronger than the observed value. The model that best matches the observed spectrum corresponds to an age of $0.5 \mathrm{Gyr}$ and solar metallicity. In Figure 3b, we illustrate that all the spectra in the inner disk be reproduced at solar metallicity for an age of $0.5 \mathrm{Gyr}$, with extinction being different at different positions of the disk. Clearly, the southwestern side of the disk is more reddened as compared to the northeastern side. Derived ages would be younger (older) by around $0.1 \mathrm{Gyr}$ if the metallicities are higher (lower) than solar values. More importantly, our spectra of inner disk regions are not consistent with mean ages greater than 0.7 Gyr. 
The outer disk spectrum suggests a population that is marginally more evolved as compared to that of the inner disk. Nevertheless, the $4000 \AA$ break, which is a signature of populations a few gigayears old, is absent. We estimate an age of $0.9 \pm 0.1$ Gyr for the outer disk. In principle, acceptable fits can be obtained by adding an old population of several gigayears, typical of galactic disks, to a population of 0.5 Gyr old. However, single SSPs give a better match to the observed spectrum than those obtained by adding an underlying old population.

As noted earlier, the NE60" spectrum corresponds to the the fossil starburst region B. The Hubble Space Telescope (HST) images of region B have revealed that this region consists of more than hundred super star clusters (SSCs). de Griis. O'Connell \& Gallagher (2001) have estimated a mean age of these SSCs to be $0.65 \pm 0.25 \mathrm{Gyr}$, with none of the clusters younger than 0.30 Gyr. Typical visual extinction values were found to be less than $1 \mathrm{mag}$. These ages were estimated based on the opticalNIR (BVIJH) spectral energy distributions of individual clusters, keeping the reddening $\mathrm{E}(\mathrm{B}-\mathrm{V})$ as a free parameter. Our spectroscopically estimated age of $0.5 \pm 0.1 \mathrm{Gyr}$, and $A_{v}=0.5 \mathrm{mag}$ are very much consistent with these photometrically estimated values. However, a reanalysis of the same HST dataset has yielded older ages $1.10 \pm 0.25$ Gyr (de Griis, Bastian \& Lamers 2003), but with mean $A_{v}<0.2$ mag. It is well-known that both ageing and reddening have the same effect on the observable photometric quantities, and hence the age and extinction cannot be derived completely independent of each other. On the other hand, in the spectroscopic method that we have adopted, the relative strengths of absorption lines fix the age, whereas the slope of the continuum fixes the extinction, thus the age, and extinction were determined independent of each other. We re-iterate that the continuum of our spectra are consistent with both the solutions (low age with high extinction or high age with low extinction), but the absorption-line ratios clearly rule out ages exceeding 0.7 Gyr for region B.

The similarity of the inner disk spectra, irrespective of whether they belong to compact knots of region B or diffuse parts of the disk, carries important clues on the star formation scenario in the disk of M 82. In the next section, we use the mass-to-light ratio in the $K$-band to establish firm constraints on the mass of the underlying old disk population, and also discuss a scenario of star formation in the disk of M 82.

\subsection{Absolute magnitude and colors}

Azimuthally averaged intensity profiles of M 82 in the NIR and the optical bands follow an exponential law expected for stellar disks of galaxies (see Figure 1). We generated the 2-dimensional exponential disk of M82 in the $K$-band based on the observed scalelength of $47^{\prime \prime}(825 \mathrm{pc})$. We then extracted the disk magnitude in the annular zone between $70^{\prime \prime}$ and $170^{\prime \prime}$, which was found to be 5.84 mag. At the distance of M 82, this corresponds to an absolute magnitude of -21.96 , without correction for extinction.

Optical and NIR colors are useful in determining the evolutionary status of the stellar systems, if the reddening in various colors can be reliably estimated. The use of multiband images allows us to obtain profiles of azimuthal and radial distribution of colors. In Figure 4, we

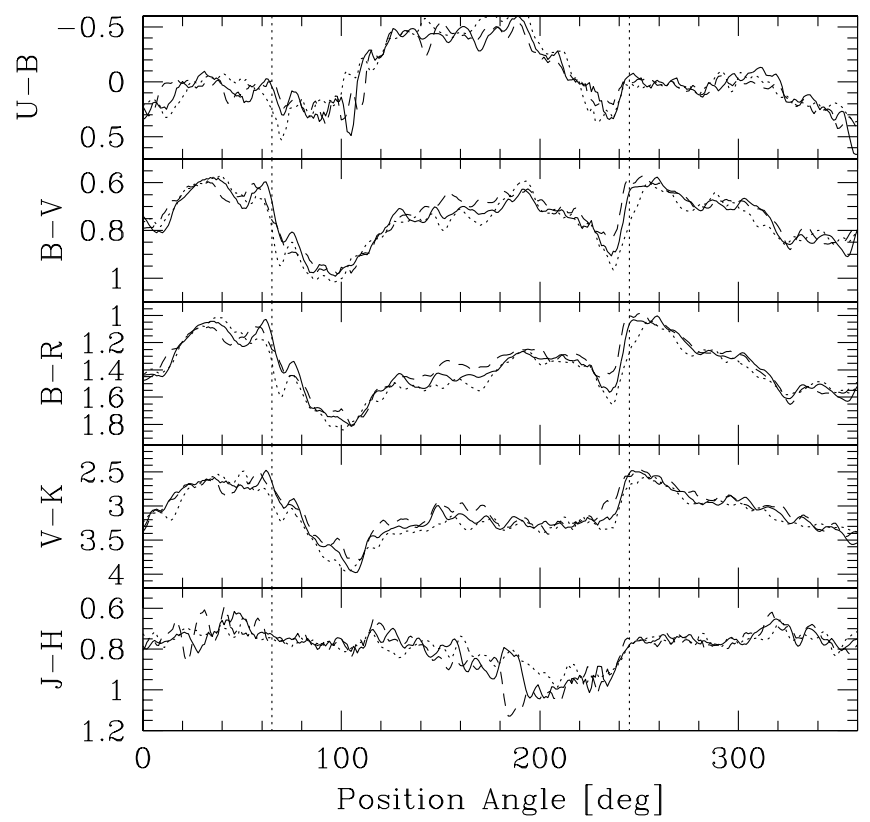

FIG. 4.- Azimuthal color profiles at radius $=110,120$ and 130" from the center. The position of the spiral arms is marked by the vertical lines. The arms, in general, are bluer than the disk. The disk colors in the azimuthal zone between $65-245^{\circ}$ is affected by the foreground dust and the superwind cone, and hence typical disk colors and their errors are derived averaging the colors between P.A. $320^{\circ}$ and $360^{\circ}$.

display the azimuthal distribution of colors in the radial zone between $90^{\prime \prime}$ and $130^{\prime \prime}$. In this radial zone, the arms are the strongest features, and hence these profiles can be used to investigate whether the results obtained from spiral-arm dominated major axis profile is applicable to the rest of the disk. In general, colors are the bluest along the major axis (dotted lines at P.A.s 65 and $245^{\circ}$ ). However, the color difference between the disk and the spiral arms at a given radius is small compared to the overall radial gradient in colors, displayed in Figure 1. Hence it seems that the results obtained from major axis spectra are representative of the entire disk.

\subsection{The Mass and mass-to-light ratio}

The rotation curve of M 82 has been derived in several studies using both stellar and gaseous tracers up to a radius of $170^{\prime \prime}$, with the intention of mapping the mass distribution in its disk. Mavall (1960) and Goetz et al. (1990) obtained a rotation curve using optical emission and absorption lines, whereas Sofue (1998) used the CO and H I lines. In all these studies, the rotation curve has been modeled to obtain the radial distribution of mass. Mavall (1960) estimated a mass of $4 \times 10^{9} \mathrm{M}_{\odot}$ in the 70-170" annulus, whereas Goetz et al. (1990) masses are $50 \%$ lower. Sofue (1998) found that most of the mass is concentrated within the central $1 \mathrm{kpc}$ radius, with the mass distribution outside this radius consistent with an exponential mass surface density profile. Significantly, there is no evidence for a dark matter halo, and hence the entire mass outside the central bar can be associ- 
ated with the stars and gas in the disk. We obtained the mass-to-light ratio ${ }^{6}$ of the disk outside a radius of $1 \mathrm{kpc}$ by combining the disk mass and the $K$-band luminosity in the same annular zone. The resulting value is $0.30 \pm 0.05 M_{\odot} / L_{K \odot}$ if we use mass estimates of Mavall (1960) and 0.15 for the mass estimates of Goetz et al. (1990). At least $15 \%$ of the disk mass in the annular zone outside $1 \mathrm{kpc}$ is in the form of atomic and molecular gas (Young \& Scoville 1984).

\subsection{Metallicity of the nebular gas and stars}

Metallic abundances of gas and stars in the starburst region of M 82 have been analyzed by Origlia et al. (2004). The cold phase gas abundances were derived using the optical and NIR nebular lines associated with the giant HII regions, whereas the stellar abundances were obtained using the NIR absorption lines that originate in the atmospheres of cool red supergiants. Since these stars live only for a few tens of million years, both the stellar and gaseous abundances are expected to represent the values present in the disk before the onset of nuclear starburst.

In Table 1, we summarize all the observed quantities for the disk, which we will use as a constraint for a detailed modeling of the star formation and chemical history of the disk of M 82 .

\section{STAR FORMATION AND CHEMICAL HISTORY OF THE DISK OF M 82}

In order to reproduce the observed quantities (metallicity enhancement, $M / L_{K}$, age and colors), we ran a number of chemical evolutionary models from the GRASIL WEB interface GALSYNTH ${ }^{7}$. In these models, gas phase metallicities of different elements are calculated over the Hubble time for a given SFH of the galaxy. At each epoch, the star formation rate (SFR) is calculated using a Schmidt law, with the exponent value of 1.0. The standard Kroupa IMF parameters are used (Kroupa et al. 1993). The current $M / L_{K}$ value is calculated as the ratio of the mass locked up in stars (both living as well as their remnants) to the luminosity of all the living stars. It is important to note that in order to reproduce the observed $M / L_{K}$ values, it is necessary to correct the model values for the mass in gas $(>15 \%)$, and the extinction in the $K$-band.

Among the observed quantities, the metallicity enhancement critically depends on the slope and the upper cut-off mass of the IMF and the burst duration, whereas the lower cut-off mass of the IMF and the age of the burst determine the $M / L_{K}$ ratios and colors. The observed set of parameters could be produced only by those models where almost all the observed stellar mass of the disk was formed by a burst lasting for only a few hundreds of million years, the burst having completely stopped around 0.5 Gyr. We ran a set of models for a wide range of input parameters. We discuss two of these models below.

\footnotetext{
6 Throughout this work, mass-to-light ratio is defined using the monochromatic $K$-band luminosity calibrated against the absolute magnitude of sun $M_{K \odot}=3.33 \mathrm{mag}$. Instead, if we use the $K$-band luminosities integrated over a standard $K$-band response curve of band width $0.35 \mu \mathrm{m}$, and express mass-to-light ratio in $\mathrm{M}_{\odot} / \mathrm{L}_{\odot}$, then the quoted values have to be multiplied by 50 .

${ }^{7}$ http://web.pd.astro.it/galsynth/index.php
}

Table 1. Observed AND MOdel Properties of the Disk of M 82

\begin{tabular}{|c|c|c|c|c|}
\hline Quantity & units & Observed & Model A & Model B \\
\hline $\begin{array}{l}M_{\text {disk }} \\
\frac{M(\text { gas })}{M(\text { disk })} \\
M_{\mathrm{K}}(\text { disk }) \\
M / L_{\mathrm{K}} \\
{[O / F e]}\end{array}$ & $\begin{array}{l}10^{9} \mathrm{M}_{\odot} \\
\ldots \\
\operatorname{mag} \\
\mathrm{M}_{\odot} / L_{\mathrm{K}_{\odot}} \\
\ldots\end{array}$ & $\begin{array}{c}<4.0 \\
>0.15 \\
-21.96 \pm 0.05 \\
<0.30 \pm 0.05 \\
0.35 \pm 0.20\end{array}$ & $\begin{array}{c}4.0 \\
0.15 \\
-21.42 \\
0.53 \\
0.11 \\
\end{array}$ & $\begin{array}{c}4.0 \\
0.15 \\
-21.95 \\
0.32 \\
0.21 \\
\end{array}$ \\
\hline
\end{tabular}

The run of the SFR of the best-fit model as a function of the age of the living stars is shown in Figure 5a. In Figure $5 \mathrm{~b}$, we show the metallicity enhancements for various metals for the standard Kroupa IMF (open circles denoted model A), along with the observed enhancements in the cold gas and stars. The corresponding values for the $M / L_{K}$ and colors are shown in the bottom panel of the figure. With the Kroupa IMF the model produces metallic enhancements which are systematically outside the observed values even after taking into account the errors in the observed metallicities. The model also produces $M / L_{K}$ values which are around $30 \%$ higher than the highest values allowed by the observations. The model colors, after reddening them with the inferred extinction values from the spectra, are consistent with the observations.

The only way we could reproduce the observed high enhancements of $\alpha$ elements and a low $M / L_{K}$ is by adopting a top-heavy IMF (model B). In this model, the slope of the Kroupa IMF for the massive stars is reduced from 1.7 to 1.4 (i.e. closer to the Salpeter slope), and the lower mass cut-off raised from 0.15 to $0.4 \mathrm{M}_{\odot}$. The resulting values are shown in Figure 5 by square symbols. This model successfully reproduces the observed trend in the metallic enhancements for all the elements except Carbon. The $M / L_{K}$ values are also very well reproduced. In fact, the agreements between the model and the observational values would be even better if we use a flatter IMF. These trends for the disk IMF are very similar to that found for the nuclear IMF by Rieke et al. (1993). The shallower IMF would imply a harder ultraviolet radiation field, contrary to what is observed for a sample of 23 starburst nuclei (Rigbv \& Rieke 2004). This apparent contradiction could be solved if the most massive stars spend most of their lifetimes embedded in dense highly extinguished regions, as suggested by Silva et al. (1998). The model quantities are compared with the observed quantities in Table 1.

In summary, models with more or less constant SFR over the Hubble time cannot reproduce neither the observed low mean age of the disk of M 82, nor the observed chemical pattern and $M / L_{K}$ values. On the other hand, observations can be only reproduced if more than $90 \%$ of the stellar mass inside the central $3 \mathrm{kpc}$ is younger than a gigayear. Hence, around 1 Gyr ago, the disk experienced an intense starburst. Furthermore, we can reproduce both the low $M / L_{K}$ value and the enhancement of the $\alpha$ elements only by a suitable modification of the Kroupa IMF. To reproduce the observed $M / L_{K}$, half a gigayear 

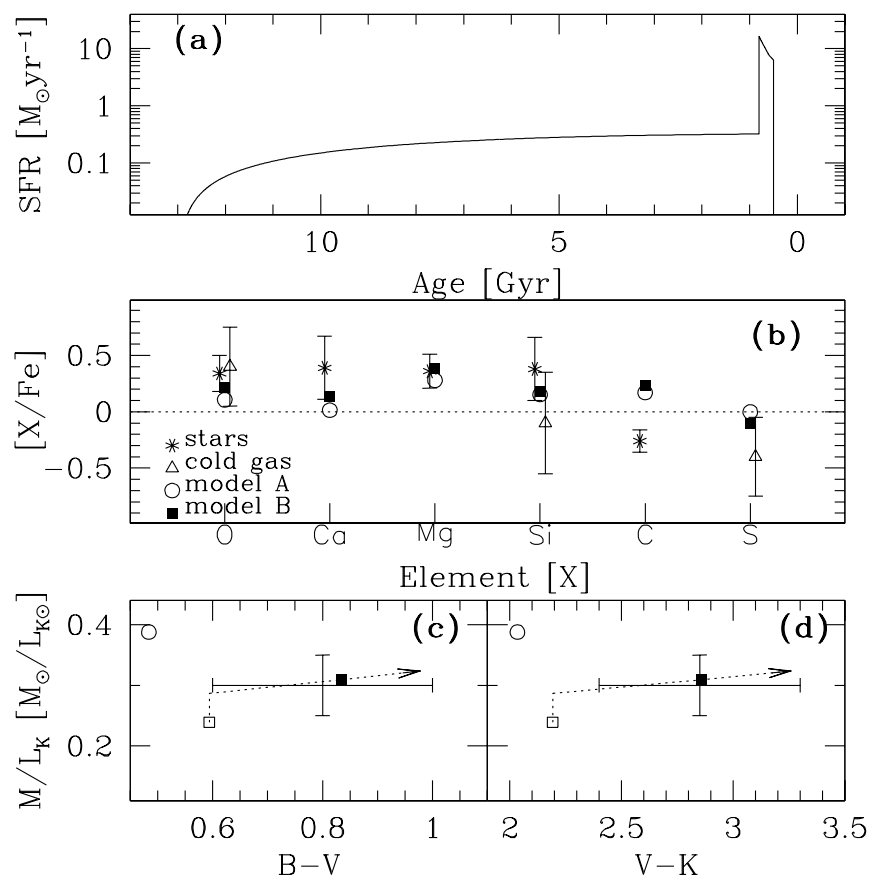

FIG. 5.- (a) Star formation History of the disk of M 82 that reproduces best the observed properties. (b) Observed stellar and nebular metallicities compared with the gas metallicities of our best-fit model. Model A and B correspond to standard and topheavy Kroupa IMFs, respectively. (c-d) $B-V$ and $V-K$ colors are plotted against the K-band mass-to-light ratio of the disk. The error bars on the colors are an indication of the observed color gradient between $80-160^{\prime \prime}$ part of the disk. Open circles and squares correspond to Model A and B, respectively, without taking into account the effect of the gas mass on the $M / L_{K}$ ratio and the interstellar extinction on the colors. In both these models, the burst is of 0.3 Gyr duration, which started around 0.8 Gyr ago. Star formation activity in the disk has ceased since then. The dotted paths indicate the locus of this point after corrections for gas content (fraction of gas mass is 15\%) and extinction (length of the arrow corresponds to $A_{v}=1.2 \mathrm{mag}$ ) are applied. Model B successfully reproduces all the observed trends.

after the burst has stopped, we are forced to truncate the lower end of the IMF at $0.4 \mathrm{M}_{\odot}$. Moreover, in order to reproduce the observed enhancement with SNIa progenitors that originate from mass accretion onto white dwarfs in binary systems (as it is adopted in our chemical model) we need to decrease the slope of the IMF of the massive stars to the Salpeter value. Thus, by combining age, luminosity and dynamical mass of the stellar populations we conclude that the disk of M 82 formed very recently and with an IMF peaked toward the massive stars. The increase in the low-mass cutoff and decrease in the slope may be due to a turbulence-enhanced ISM, probably as a result of interaction or/and the starburst episode itself.

\section{EVOLUTIONARY STATUS OF M 82}

Disks of normal spiral galaxies are characterized by star formation that is continuing un-interrupted over the Hubble time (Kennicutt et al. 1994). M 82 differs in two ways from these normal disks: (1) luminosity weighted age of the disk is only $0.5 \mathrm{Gyr}$, which is an order of magnitude younger, and (2) there is no present star for- mation in the disk. M82 also differs from the normal galaxies in the relations between global parameters, it is too bright and also metal rich for its low mass value (Karachentsev et al. 2004). The origin of these peculiarities of M 82 could be understood with our inferred $\mathrm{SFH}$, in which almost the entire observed stellar mass was formed in a short-duration burst, rather than in a continuous manner. In our best-fit model, the diskwide burst started around $0.8 \mathrm{Gyr}$ ago, which is consistent with the interaction scenario proposed by Sofue (1998). It is interesting to note that a number of interacting galaxies also show disk-wide star formation at present: examples being NGC 4038/9, Arp 299 and NGC 4676 (Hibbard \& Yun 1999; Mirabel et al. 1998; $\mathrm{Xu}$ et al. 2000). This kind of widespread star formation in galactic disks is probably induced by shocks generated in the interstellar medium following the interaction (Barnes 2004).

Mihos (1999) discussed pre-collisional galaxy properties that favors star formation in the disk, before the burst is turned on in the center. He found that the precollisional galaxy should be a normal high-surface brightness galaxy with a big bulge or a gas-rich low surface brightness galaxy (LSB). If the pre-collisional M 82 had a big bulge, it would have survived the interaction (Sofue 1998). However, the observed bulge of M 82 is small, consequently, pre-collisional M 82 should have been a gasrich LSB galaxy. This is in agreement with our proposed SFH. It could be that either M 82 was a gas-rich LSB galaxy before the interaction, or that it acquired vast amounts of gas during the interaction. Below we discuss three scenarios that are consistent with the present appearance of M 82 .

\subsection{Kinematically disturbed normal galaxy}

The most important distinguishing characteristic of M 82 is its low value of M/L ratio as compared to that of normal galaxies. Is this because of an underestimation of its mass? The kinematics of the galaxy could have been disturbed because of interaction resulting in possible underestimation of its mass. Blue-band spectra would look identical even if we add upto a factor of 3-5 more mass in stars older than 5 Gyr as compared to the mass in stars 10 times younger. However, the presence of such an amount of old stars would have contributed to the enrichment of $\mathrm{Fe}$, without the corresponding enrichment of $\alpha$ elements, thus resulting in low values of $[\alpha / \mathrm{Fe}]$. Thus the observed enhancement of $\alpha$ elements indicates that the galaxy really lacks a significant population of stars older than 1 Gyr.

\subsection{Rejuvenated star formation in a stripped stellar disk}

In the scenario proposed by Sofue (1998), much of the disk and halo of M 82 were stripped off during its interaction with the members of the M81 group. However, none of the observational studies so far have detected the stripped dark matter halo, containing the old disk. It would be interesting to carry out a search for such a system in the M 81 group. Even in this scenario, the gas and stars in the central kiloparsec region are not stripped, and hence the observed $\alpha$ enhancement of the nuclear gas requires vast amounts of star formation, and inflow of the metal-enriched material, after the original 
disk has been lost. In other words, the SFH we have inferred is consistent with this scenario, and hence M 82 is in the process of re-building its stellar disk.

\subsection{A nearby galaxy in formation?}

Analysis of galaxies at redshifts up to 1 support a morphological evolution over the past few gigayears. The catalyst that drives the morphological evolution is the major mergers between galaxies. During the merging process galaxies grow both in size and luminosity. Data suggest that at least $50 \%$ of the nearby disk galaxies are formed through this scenario (Hammer et al. 2005). The most important ensemble of galaxies that are thought to be caught in the formation process are the luminous blue compact galaxies (LBCGs) seen between redshifts $0.2-1.0$. The characteristic signature of these galaxies is the enhanced star formation in their nuclear regions. In a recent study, Noeske et al. (2006) have characterized the properties of the stellar disk surrounding the compact nucleus, and found that they are, in general, short in scalelength as compared to the scalelengths of local galaxies.

M 82 resembles these LBCGs in having high luminosity, short scalelength, a nuclear starburst and low mass. Moreover, interaction is responsible for all the observed characteristics in M 82. Thus in M 82, we may be witnessing stars being formed for the first time in its disk, probably in the debris left behind in the interaction. In other words, M 82 could be a nearby galaxy in formation.

\section{SUMMARY}

We used spectrophotometric, photometric and dynamical data of the inner $\approx 3^{\prime}(3 \mathrm{kpc})$ radius of $\mathrm{M} 82$, to study the star formation history in the inner disk of M 82. The optical diameter (measured at $\mu_{B}=25 \mathrm{mag} \mathrm{arcsec}^{-2}$ ) of M 82 is around $13^{\prime}$, and hence our analysis pertains to the inner half of the disk. We find that a large fraction of the inner disk is as young as $0.5 \mathrm{Gyr}$. The models that best reproduce the above observations indicate that the star formation in the disk happened in a burst of $0.3 \mathrm{Gyr}$ duration, that ended around $0.5 \mathrm{Gyr}$ ago. The short duration of the burst is not enough to reproduce both the enhancement of the $\alpha$ elements and the low value of the $M / L_{K}$ ratio, and a suitable top-heavy IMF must be invoked. It is possible that the star formation started in the whole disk about 0.8 Gyr ago, induced by shocks in the interstellar medium following the interaction with members of M 81 group, and now continues only in the central regions.

We are grateful to L. Silva and G. L. Granato for providing us their chemical evolutionary model before its publication. We thank Abelardo Mercado for carrying out some of the spectroscopic observations used in this study. Suggestions by an anonymous referee have greatly helped in improving the presentation of this manuscript. This work was partly supported by the CONACyT (Mexico) projects 39714-F and G28586E. This research has made use of the NASA/IPAC Extragalactic Database, which is operated by the Jet Propulsion Laboratory, California Institute of Technology, under contract with the National Aeronautics and Space Administration.

\section{REFERENCES}

Barnes, J. E. 2004, MNRAS, 350, 798

Bressan, A., Fagotto, F., Bertelli, G., \& Chiosi, C. 1993, A\&AS, 100,647

Bressan, A., Granato, G. L., \& Silva, L. 1998, A\&A, 332, 135

Cardelli, J. A., Clayton, G. C., \& Mathis, J. S. 1989, ApJ, 345, 245

Elvius, A. 1972, å, 19, 193

Fagotto, F., Bressan, A., Bertelli, G., \& Chiosi, C. 1994a, A\&AS, 105,29

Fagotto, F., Bressan, A., Bertelli, G., \& Chiosi, C. 1994b, A\&AS, 104,365

Freedman, W. L. et al. 1994, ApJ, 427, 628

Gaffney, N. I, Lester, D. F, \& Telesco, C. M. 1993, ApJ, 407, 57

Goetz, M., McKeith, C.D., Downes, D., \& Greve, A. 1990, A\&A, 240,52

de Grijs, R., O'Connell, R. W., \& Gallagher, J. S. 2001, AJ, 121, 768

de Grijs, R., Bastian, N., \& Lamers, H. J. G. L. M. 2003, MNRAS, 340,197

Hammer, F., Flores, H., Elbaz, D., Zheng, X. Z., Liang, Y. C., \& Cesarsky, C. 2005, A\&A, 430, 115

Hibbard, J. E., \& Yun, M. S. 1999, AJ, 118, 162

Holmberg, E. 1950, Lund Medd. Astron. Obs. Ser. II, 128, 1

Ichikawa, T., Yanagisawa, K., Itoh, N., Tarusawa, K., van Driel, W., \& Ueno, M. 1995, AJ, 109, 2038

Jacoby, G. H., Hunter, D. A., \& Christian, C. A. 1984, ApJS, 56, 257

Karachentsev, I. D., Karachentseva, V. E., Huchtmeier, W. K., \& Makarov, D. I. 2004, AJ, 127, 2031

Kennicutt, R. C., Tamblyn, P., \& Congdon, C. E. 1994, ApJ, 435, 22
Kroupa, P., Tout, C. A., \& Gilmore, G. 1993, MNRAS, 262, 545

Marcum, P. M., et al. 2001, ApJS, 132, 129

Mayall, N. U. 1960, Ann. d'Ap., 23, 344

Mayya, Y. D., Carrasco, L., \& Luna, A. 2005, ApJ, 628, L33

Mihos, J. C. 1999, IAU Symp. 186: Galaxy Interactions at Low and High Redshift, 186, 205

Mirabel, I. F., et al. 1998, A\&A, 333, L1

Noeske, K. G., Koo, D. C., Phillips, A. C., Willmer, C. N. A. Melbourne, J., Gil de Paz, A., \& Papaderos, P. 2006, ApJ, 640, L143

O'Connell, R. W., \& Mangano, J. J. 1978, ApJ, 221, 62

Origlia, L., Ranalli, P., Comastri, A., \& Maiolino, R. 2004, ApJ, 606,862

Rieke, G. H., Lebofsky, M. J., Thompson, R. I., Low, F. J., \& Tokunaga, A. T. 1980, ApJ, 238, 24

Rieke, G. H., Loken, K., Rieke, M. J., \& Tamblyn, P. 1993, ApJ, 412, 99

Rigby, J. R. \& Rieke, G. H. 2004, ApJ, 606, 237

Silva, L., Granato, G. L., Bressan, A., \& Danese, L. 1998, ApJ, 509,103

Sofue, Y. 1998, PASJ, 50, 227

Telesco, C. M., Joy, M., Dietz, K., Decher, R., \& Campins, H. 1991, ApJ, 369, 135

Young, J.S., \& Scoville, N. Z. 1984, ApJ, 287, 153

Yun, Min S., Ho, Paul T. P., \& Lo, K. Y. 1994, Nature, 372.530

Xu, C., Gao, Y., Mazzarella, J., Lu, N., Sulentic, J. W., \& Domingue, D. L. 2000, ApJ, 541, 644 